\title{
Disadvantages of Control Chart in Printing Quality Control over Solder Paste and Strategies for Improvement
}

\author{
Chen Peng \\ School of Mechanical Engineering, Nanjing University of Science and Technology \\ 200 Xiaolinwei, Nanjing 210094, China \\ School of Mechatronics, Nanjing College of Information and Technology \\ 99 Wenlan Road, Xianlin, Nanjing 210046, China \\ Tel: 86-25-8584-2287 E-mail: njxinfumm@hotmail.com \\ Kenny Liu \\ Norautron Suzhou Co.,Ltd \\ No.35 Dong Jing Economic Development Co.,Ltd \\ E-mail: bigkennyliu@sina.com \\ Xiaohui Gu \\ School of Mechanical Engineering, Nanjing University of Science and Technology \\ Tel: 86-25-8430-3233 E-mail: gxiaohui@mail.njust.edu.cn
}

\begin{abstract}
Control chart is one kind of the most common and effective tools for SPC quality control, however, it is not infallible for ever. The results of actual data processing have proved that the conventional control chart may educe the opposite conclusions to other evaluating methods in some special cases. For solving the above problems, we use Bayes theory to introduce proper 'correction coefficient' to amend the control parameters so that the control limit is more accurate and the judgment is more reasonable and reliable.
\end{abstract}

Keywords: Control chart, SPC, CPI, Correction coefficient, Bayes theory

\section{Introduction}

Statistical Process Control (SPC) is a process control tool making use of Mathematical Statistics. The control chart is a statistical tool controlling the products quality in the process of manufacture, which is also one kind of the most common and effective tools for SPC quality control.

During the application of control chart, its ordinate axis is set as the quality characteristic value, whose scales are divided by the processing variation; its horizontal axis is the sampling time or sample number of measured products, which is drawn on the figure according to the precedence, as shown in figure 1. There are three straight transverse lines in the control chart, the middle one of which is Center Line (CL) protracted by blue solid. The upper line is called Upper Control Limit (UCL) and the lower one is called Lower Control Limit (LCL), which are often represented by red dash lines denoting the acceptable arrange of variation. The quality characteristics of the actual products are shown by black lines.

Once the control chart is evaluated that, 'the quality management begins with control chart and also ends with control chart.' The control chart changes the quality control mode from post-checkout to prevention, which provides a wide prospect for guaranteeing the quality of products, reducing production costs, enhancing productivity, so it has been applied widely in many countries. However, control chart is not infallible for ever and the conventional control chart may educe the opposite conclusions to other evaluating methods in some special cases.

\section{Process Capability Indices}

Process Capability Indices (CPI) is used to indicate the capability of assuring the quality of production in the normal condition of process, which means that Man, Machine, Material, Technique, Measurement and Environment are fully standardized and in the stable state. CPI is also named Capability Index of Process (CPK) and the corresponding calculation formula is shown as follows: 
$\mathrm{CPI}=$ Technical Specification Requirements/ Process Capability

When the distributing centre coincides with the tolerance center, CPI is marked as $C_{p}$, otherwise, CPI is marked as $C_{p k}$, which is shown as figure 2 .

The calculation of CPI can be divided into 4 types (Zhao, Yandong. 2005), which is shown as table 1.

The process quality can be divided into 5 grades according to $C_{p k}$, which is shown as table 2 .

\section{Contradiction between control chart and CPI}

If we get enough samples in the machining, the samples will follow the normal distribution $\bar{X} \sim N\left(\mu, \frac{\sigma^{2}}{n}\right)$, where $\mu_{\text {and }} \sigma^{2}$ are the average and variance of population, respectively. The CL, UCL and LCL of $\bar{X}$ control chart are shown below according to the $3 \sigma$ theory:

$$
\left\{\begin{array}{l}
U C L_{x}=\mu+3 \sigma \\
C L_{x}=\mu \\
L C L_{x}=\mu-3 \sigma
\end{array}\right.
$$

When the measured data are monitored using the above formula, we can draw a control chart shown in figure3. From the criterion of control chart(Liu Keneng, Peng Chen and Fu Geyan. 2009), there are two abnormal points A \& C between UCL and LCL in figure 3, which denotes that some problems arise in machining and we have to stop machines to check. If now we calculate $C_{p k}$, the value may be higher and even more than grade 2 . We can come to the conclusion through testing the productions.

In fact, in the actual activity of sampling, the samples can not be infinite even very small in some cases, so the boundary of control chart made according to the normal distribution may appear some deviation, which will directly affect the judgment result. For figure 3, when we make necessary correction for the original chart according to some other known conditions, the factual control boundary is shown as UCL' \& LCL' and the abnormal point only contains point B in this case.

Therefore, this paper will analyze the reasonable corrective method of controlling the boundary of chart and CPI as well as decision-made owned by human beings to obtain more reliable and truthful quality control statistic theory and measurement.

\section{The correction of control chart boundary}

The applied basis of control chart is based on the quality index of obtained data totally obeying to the normal distribution, where the deviation existing in the parameters of $N\left(\mu, \sigma^{2}\right)$ are not large can be known that the parameters $\mu$ and $\sigma^{2}$ separately obey some prior distributions $F(\mu)$ and $G\left(\sigma^{2}\right)$. Because the estimation is deduced statistically, it is possible for deviations existing which may cause loss. The two characteristics of the actual problem just accord with the requirement of Bayes analysis. The Bayes analysis suppose the distributing parameters of indexes contain the prior distribution, so the parameters can be deduced statistically according to the prior distribution and current samples. In the condition that a reasonable prior distribution with small variance is chosen, the Bayes estimation of small samples can improve the traditional estimation well. Therefore, we can obtain the Bayes estimation of the index parameters of current productions fully using the smaller samples of past productions and current ones.

The parameter $\mu$ to be estimated is a random variable during Bayesian analysis. People can manually determine the probability density $\varphi(\mu)$ of parameter $\mu$ according to the past experiences, which is named the prior distribution of $\mu$. Supporting $x_{1}, x_{2}, \ldots, x_{n}$ are a sample from the total samples $\mathrm{X}$, the sampling result is $\zeta$. Then the prior distribution density $\varphi(\mu)$ of $\mu$ is corrected using Bayes method according to the information provided by $\zeta$ to obtain the posterior distribution density $\varphi^{\prime}(\mu)$. The formula (2) is shown as follows:

$\varphi^{\prime}(\mu) \Delta \mu \approx \frac{P\left(\zeta \mid \mu_{i}<\mu \leq \mu_{i}+\Delta \mu\right) \bullet \varphi\left(\mu_{i}\right) \Delta \mu}{\sum_{k} P\left(\zeta \mid \mu_{i}<\mu \leq \mu_{i}+\Delta \mu\right) \bullet \varphi\left(\mu_{i}\right) \Delta \mu}$ 
When $\Delta \mu \rightarrow 0$, the limit of the above formula is

$$
\varphi^{\prime}(\mu)=\frac{P(\zeta \mid \mu) \bullet \varphi(\mu)}{\int_{-\infty}^{+\infty} P(\zeta \mid \mu) \bullet \varphi(\mu) d \mu}
$$

All the samples obey the law of $X \sim N\left(\mu, \sigma^{2}\right)$, so

$$
P(\zeta \mid \mu)=\prod_{k=1}^{n} \frac{1}{\sigma \sqrt{2 \pi}} e^{\frac{-\left(x_{i}-\mu\right)^{2}}{2 \sigma^{2}}} \cdot d x \Delta \prod_{k=1}^{n} N_{\mu}\left(x_{k}, \sigma^{2}\right) d x
$$

In formula (4), $N_{\mu}\left(x_{k}, \sigma^{2}\right)$ denotes the distribution density of normal random variable of some observed value $x_{k}$, where $\mu$ and $\sigma^{2}$ are respectively the average and variance of total samples.

$$
\begin{gathered}
\varphi^{\prime}(\mu)=\frac{\left[\prod_{k=1}^{n} N_{\mu}\left(x_{k}, \sigma^{2}\right)\right] \bullet \varphi(\mu)}{\int_{-\infty}^{+\infty}\left[\prod_{k=1}^{n} N_{\mu}\left(x_{k}, \sigma^{2}\right)\right] \bullet \varphi(\mu) d \mu}=\frac{N_{\mu}\left(\bar{x}, \sigma^{2} / n\right) \bullet \varphi(\mu)}{\int_{-\infty}^{+\infty} N_{\mu}\left(\bar{x}, \sigma^{2} / n\right) \bullet \varphi(\mu) d \mu} \\
=m \bullet N_{\mu}\left(\bar{x}, \sigma^{2} / n\right) \bullet \varphi(\mu) \quad-\bar{x}=\frac{1}{n}\left(x_{1}+x_{2}+\ldots+x_{n}\right) \\
\text { Where } m=\left(\int_{-\infty}^{+\infty} N_{\mu}\left(\bar{x}, \sigma^{2} / n\right) \bullet \varphi(\mu) d \mu\right)^{-1} \text { is constant and }
\end{gathered}
$$

After we get the posterior distribution density $\varphi^{\prime}(\mu)$ of parameter $\mu$ made from $x_{1}, x_{2}, \ldots, x_{n}$, then we can combine the error because of estimating $\mu$ using $\bar{X}$ and the implicit random variable $\mathrm{X}$, which can be reckoned in the random variable $\mathrm{X}$ using the total probability formula. Now, the distribution density formula of random variable $\mathrm{X}$ is as follows:

$f_{x}(x)=\int_{-\infty}^{+\infty} f_{x}(x \mid \mu) \varphi^{\prime}(\mu) d \mu$

When no data or information about $\mu$ is provided, from the limit theory in probability we know that: $\mu$ is uniform distribution and $\varphi(\mu)$ almost equals to 1 everywhere (the probability of $\varphi(\mu) \neq 1$ is 0 , which is the event with 0 probability). Further more, $\varphi^{\prime}(\mu)$ is the distribution density of random variable and $m$ should be 1 almost everywhere (the probability of $m \neq 1$ is 0 , which is the event with 0 probability), so

$\varphi^{\prime}(\mu)=N_{\mu}\left(\bar{x}, \sigma^{2} / n\right) \bullet \varphi(\mu)$

Substitute formula (7) into the formula (6), then

$f_{x}(x)=\int_{-\infty}^{+\infty} N_{x}\left(\mu, \sigma^{2}\right) \bullet N_{\mu}\left(\bar{x}, \sigma^{2} / n\right) d \mu=N_{x}\left(\bar{x}, \sigma^{2}+\sigma^{2} / n\right)$

This means $X \sim N\left(\bar{x}, \sigma^{2}+\sigma^{2} / n\right)$. So according to "3 $\sigma$ Principle", the control boundary of $\mathrm{X}$ is:

$$
\left\{\begin{array}{l}
U C L_{x}=\bar{X}+3 \sqrt{\sigma^{2}+\sigma^{2} / n} \\
C L_{x}=\bar{X} \\
L C L_{x}=\bar{X}-3 \sqrt{\sigma^{2}+\sigma^{2} / n}
\end{array}\right.
$$

Thus, comparing with formula (1), when there is no information about $\mu$, the controlling center line $C L_{x}$ does 
not change, but the locations of $U C L_{x}$ and $L C L_{x}$ change.

On the other hand, if the data or information about $\mu$ has existed, which means that $X \sim N\left(\mu_{1}, \sigma_{1}^{2}\right)$ (where $\mu_{1}$ and $\sigma_{1}^{2}$ are the known constants)has been known, the control boundary of $\mathrm{X}$ is:

$\left\{\begin{array}{l}U C L_{x}=\mu_{2}+3 \sqrt{\sigma^{2}+\sigma_{2}^{2}} \\ C L_{x}=\mu_{2} \\ L C L_{x}=\mu_{2}-3 \sqrt{\sigma^{2}+\sigma_{2}^{2}}\end{array}\right.$

Where $\sigma_{2}^{2}=\left(\sigma_{1}^{2} \bullet \sigma^{2} / n\right) /\left(\sigma_{1}^{2}+\sigma^{2} / n\right), \mu_{2}=\left(\bar{x} \bullet \sigma_{1}^{2}+\mu_{1} \bullet \sigma^{2} / n\right) /\left(\sigma_{1}^{2}+\sigma^{2} / n\right)$

\section{Illustration}

Now, we take some solder paste volume test results as an example shown in table 3, and the test frequency is 2 panels/hour.

$\bar{x}-R$ control chart are shown in figure 4,5 and 6 respectively.

Based on the data $C_{p k}$ listed in table 3, we calculate the following:

$T_{u}=18.95, T_{l}=10.20, T=T_{u}-T_{l}=18.95-10.20=8.75, \quad \sigma=1.0538$

So,

$$
C_{p}=\frac{T}{6 \sigma}=\frac{8.75}{6 \times 1.0538}=1.384
$$

As $\overline{\bar{X}}=14.68$ is different with $T_{m}=\left(T_{l}+T_{u}\right) / 2=14.575$, therefore, there is some deviation in the process:

$$
\begin{aligned}
& k=\frac{\left|\mu-T_{m}\right|}{T / 2}=0.024 \\
& C_{p k}=(1-k) C_{p}=1.35
\end{aligned}
$$

From figure 4 , we know that the control chart R is not abnormal. However, the $23^{\text {rd }}$ point is out of LCL in figure 5 , which is considered abnormal in the process according to the conventional judgment method. It is found that the value of $C_{p k}$ is higher to 1.35 through calculation. From figure 2, the corresponding conclusion is "the process ability is good enough" and what we should do is "lower requirements for raw material, simplifying the quality testing, adopting sampling tests or decreasing test frequency". The contrary results prove the judgment for traditional control chart is not accuracy.

From figure 4 and 6, there are no abnormal conditions in control charts $R$ and $\bar{X}$. The value of $C_{p k}$ is 1.35 , the corresponding conclusion is "the process ability is good enough" and what we should do is "lower requirements for raw material, simplifying the quality testing, adopting sampling tests or decreasing test frequency". These two conclusions of judgment match each other well, which denotes that the corrected method is more reliable and truthful for the real production.

\section{Conclusion}

This paper takes the common control chart $\bar{x}-R$ as an example and discussed the advantages in the process of application. When the boundary of control chart is determined, which only depends on the sampling data to estimate and not consider the former conditions or other interfere or just concerns the information of total and samples but ignores the acquired or existed information, it is usually found that the conclusion may conflict with other judgment indexes. Thus, to solve the above problems, we can use Bayes theory to introduce the proper "correction coefficient" to correct the control parameters, which can high the accuracy of control limits and make the conclusion more reasonable, reliable and believable. 


\section{References}

Liu Keneng, Peng Chen and Fu Geyan. (2009). The application of SPC in Printing Quality Control over Solder Paste. Siwang Press.

Zhang, Liandi and Han, Xiaoyou.(1999). An Application of Bayesian Method to Correct Quality Control Limits. Journal of Mathematical Research and Exposition, 312-314.

Zhao, Yandong. (2005). Study of Statistical Process Control (SPC) Application in Printing Quality Management. Wuhan University, 15, 20-25.

Table 1. Calculations of CPI

\begin{tabular}{|c|c|c|c|}
\hline No. & Types & Formulas & Comments \\
\hline (1) & $\begin{array}{c}\text { No skewing in } \\
\text { process }\end{array}$ & $C_{p}=\frac{T}{6 \sigma}$ & $\begin{array}{c}\text { Upper and lower limits exist when } \\
\mu=T_{m}\end{array}$ \\
\hline (2) & Skewing in process & $C_{p k}=(1-k) C_{p}$ & $\begin{array}{c}\text { Upper and lower limits exist } \\
\text { when } \mu \neq T_{m} \text { and } \mathrm{k} \text { is called } \\
\text { offset coefficient. }\end{array}$ \\
\hline (3) & $\begin{array}{c}\text { Only upper limit } T_{u} \\
\text { exits, } \mathrm{X}<T_{u}\end{array}$ & $C_{p}(u)=\frac{T_{u}-\mu}{3 \sigma}$ & \\
\hline (4) & $\begin{array}{c}\text { Only lower limit } T_{l} \\
\text { exits, } \mathrm{X}>T_{l}\end{array}$ & $C_{p}(l)=\frac{\mu-T_{l}}{3 \sigma}$ & \\
\hline
\end{tabular}

Note that: Supposing the sample quality characteristic value fits normal distribution $X \sim N\left(\mu, \sigma^{2}\right)$, and the specification for $X$ is $\left(T_{l}, T_{u}\right)$. Here, central value $T_{m}=\left(T_{l}, T_{u}\right) / 2$ while tolerance $\mathrm{T}=\left(T_{u}-T_{l}\right)$.

Table 2. Different CPI for Different Process Ability

\begin{tabular}{|l|l|l|l|l|}
\hline Range & Class & $\begin{array}{l}T_{u}-\mu \text { or } \\
\left(\mu-\mathrm{T}_{1}\right)\end{array}$ & Judgment & Comments \\
\hline$C_{p k} \geq 1.67$ & $\begin{array}{l}\text { Supper- } \\
\text { fine }\end{array}$ & $\geq 5 \sigma$ & $\begin{array}{l}\text { Too high } \\
\text { process } \\
\text { ability }\end{array}$ & $\begin{array}{l}\text { Shrink key projects tolerance range for higher } \\
\text { product quality; or lower equipments accuracy or } \\
\text { wider fluctuation range for higher efficiency or lower } \\
\text { cost. }\end{array}$ \\
\hline $1.33<C_{p k}<1.67$ & Grade 1 & $4 \sigma \sim 5 \sigma$ & $\begin{array}{l}\text { Enough } \\
\text { process } \\
\text { ability }\end{array}$ & $\begin{array}{l}\text { Wider fluctuation range for non-critical } \\
\text { project; or lower requirements for raw } \\
\text { material; or use sampling test or lower } \\
\text { test frequency to simplify quality } \\
\text { inspection. }\end{array}$ \\
\hline $1<C_{p k} \leq 1.33$ & Grade 2 & $3 \sigma \sim 4 \sigma$ & $\begin{array}{l}\text { Acceptable } \\
\text { process } \\
\text { ability }\end{array}$ & $\begin{array}{l}\text { Control chart or other methods must be } \\
\text { used to monitor or control abnormal } \\
\text { change, and inspect products in normal } \\
\text { way. }\end{array}$ \\
\hline $0.67 \leq C_{p k} \leq 1$ & Grade 3 & $2 \sigma \sim 3 \sigma$ & $\begin{array}{l}\text { Insufficiency } \\
\text { process } \\
\text { ability }\end{array}$ & $\begin{array}{l}\text { Analyze the reasons for large scatteration } \\
\text { and take measures for improvement. On } \\
\text { condition that it does not affect the } \\
\text { quality of production, strengthen quality } \\
\text { inspection and its frequency. }\end{array}$ \\
\hline$C_{p k}<0.67$ & Grade 4 & $<2 \sigma$ & $\begin{array}{l}\text { Unacceptable } \\
\text { process } \\
\text { ability }\end{array}$ & $\begin{array}{l}\text { Usually, stop working to find the reasons } \\
\text { and improve process to improve } C_{p} . \\
\text { Otherwise, seek out the unaccepted ones. }\end{array}$ \\
\hline
\end{tabular}


Table 3. Data for Solder Paste Volume Sampling

\begin{tabular}{|c|c|c|c|c|c|c|c|c|c|c|c|}
\hline Process & Machine & Parameter & Gage & \multicolumn{2}{|c|}{$\begin{array}{c}\text { USL } \\
\left(10^{7} \mathrm{um}^{3}\right)\end{array}$} & $\begin{array}{c}\text { LSL } \\
\left(10^{7} \mathrm{um}^{3}\right)\end{array}$ & $\begin{array}{l}\text { Sampl } \\
\text { eSize }\end{array}$ & $\begin{array}{c}\text { Freque } \\
\text { ncy }\end{array}$ & $\begin{array}{l}\text { Model } \\
\text { name }\end{array}$ & \multicolumn{2}{|c|}{$\begin{array}{l}\text { SPC chart } \\
\text { number }\end{array}$} \\
\hline Printing & DEK & Volume & KY3030 & 18.95 & & 0.20 & 8 & $\begin{array}{c}\text { 2panels } \\
\mathrm{hr}\end{array}$ & Q2686 & Q2686 & C49_1 \\
\hline \multirow{2}{*}{ Time } & \multirow{2}{*}{$\begin{array}{l}\text { Operat } \\
\text { or No. }\end{array}$} & \multirow{2}{*}{$\begin{array}{l}\text { Average } \\
(\bar{X})\end{array}$} & \multirow{2}{*}{$\begin{array}{c}\text { Range } \\
\text { (R) }\end{array}$} & \multicolumn{8}{|c|}{ Samples of Measurements } \\
\hline & & & & 1 & 2 & 3 & 4 & 5 & 6 & 7 & 8 \\
\hline $19: 50$ & 4073 & 14.98 & 2.45 & 16.01 & 15.32 & 15.38 & 15.97 & 13.65 & 13.56 & 15.62 & 14.32 \\
\hline $20: 50$ & 4073 & 15.09 & 3.43 & 15.53 & 12.54 & 15.38 & 15.97 & 15.53 & 15.13 & 15.13 & 15.53 \\
\hline $21: 50$ & 4073 & 15.17 & 2.15 & 15.34 & 15.13 & 15.53 & 15.53 & 15.53 & 13.38 & 15.38 & 15.53 \\
\hline $22: 50$ & 4073 & 14.34 & 3.00 & 13.68 & 14.35 & 12.54 & 15.38 & 12.54 & 15.13 & 15.53 & 15.54 \\
\hline $20: 45$ & 4073 & 14.11 & 3.09 & 12.45 & 13.26 & 14.23 & 13.35 & 15.38 & 15.12 & 15.54 & 13.55 \\
\hline $21: 45$ & 4073 & 15.04 & 2.99 & 12.55 & 15.13 & 15.53 & 15.36 & 15.54 & 15.13 & 15.53 & 15.54 \\
\hline $22: 45$ & 4073 & 14.28 & 2.99 & 12.54 & 15.13 & 13.52 & 15.53 & 15.15 & 12.88 & 15.11 & 14.35 \\
\hline $0: 50$ & 8815 & & & 13.12 & & & 13.12 & 14.12 & 15.54 & 15.39 & 15.64 \\
\hline $1: 50$ & 8815 & 15.01 & 2.50 & 15.53 & 15.16 & 15.34 & 15.26 & 13.95 & 15.69 & 15.84 & 13.34 \\
\hline $2: 50$ & 8815 & 14.00 & 3.26 & 14.02 & 13.55 & 12.54 & 15.80 & 12.65 & 15.53 & 13.22 & 14.65 \\
\hline $3: 50$ & & & & 16.01 & & 15.54 & 14.65 & 15.17 & 15.16 & 15.12 & 14.00 \\
\hline 18:15 & 4073 & 15.28 & 1.18 & 15.13 & 15.28 & 15.38 & 15.53 & 15.53 & 15.53 & 14.35 & 15.53 \\
\hline 19:15 & 4073 & 14.17 & 3.48 & 12.54 & 13.25 & 14.02 & 13.25 & 16.02 & 13.36 & 15.53 & 15.39 \\
\hline $20: 15$ & & & & 12.54 & & 12.88 & 15.34 & 13.66 & 14.25 & 15.26 & 15.84 \\
\hline $21: 15$ & 4073 & 14.33 & 2.99 & 13.55 & 14.55 & 13.66 & 14.55 & 12.54 & 15.13 & 15.13 & 15.53 \\
\hline $22: 15$ & 4073 & & 2.15 & 15.34 & 15.13 & 15.53 & 15.53 & 15.53 & 13.38 & 15.38 & 15.53 \\
\hline $22: 50$ & 8815 & & & 13.68 & 14.35 & 15.54 & 15.38 & 14.39 & 15.13 & 15.53 & 15.54 \\
\hline $0: 50$ & 8604 & 14.94 & 2.19 & 15.54 & 15.53 & 15.53 & 13.35 & 15.38 & 15.12 & 15.54 & 13.55 \\
\hline $1: 50$ & 8604 & & & 13.00 & 12.54 & 12.66 & 14.05 & 15.54 & 15.13 & 15.53 & 15.54 \\
\hline $2: 50$ & 8604 & 15.18 & 1.18 & 15.26 & 15.13 & 15.36 & 15.53 & 15.15 & 15.53 & 15.11 & 14.35 \\
\hline $3: 50$ & 8815 & 15.15 & 2.60 & 15.54 & 14.67 & 13.34 & 15.16 & 15.94 & 15.54 & 15.39 & 15.64 \\
\hline $4: 50$ & 8815 & & 3.29 & 13.25 & 14.25 & 13.65 & 12.55 & 13.95 & 15.69 & 15.84 & 13.34 \\
\hline $5: 50$ & 8815 & 13.64 & 2.99 & 12.55 & 13.66 & 13.65 & 12.58 & 12.88 & 13.65 & 15.54 & 14.65 \\
\hline $7: 50$ & 8604 & 15.01 & 1.28 & 14.64 & 14.26 & 15.54 & 14.65 & 15.17 & 15.16 & 15.12 & 15.53 \\
\hline $8: 50$ & 8604 & 15.23 & 1.18 & 15.13 & 15.28 & 15.38 & 15.53 & 15.12 & 15.53 & 14.35 & 15.53 \\
\hline 9:50 & 8604 & 13.83 & 3.31 & 12.54 & 13.02 & 12.68 & 15.53 & 15.85 & 12.57 & 13.02 & 15.39 \\
\hline $10: 50$ & 8604 & 15.34 & 1.02 & 15.00 & 14.82 & 15.53 & 15.34 & 15.36 & 15.53 & 15.26 & 15.84 \\
\hline $21: 10$ & 9941 & 15.47 & 0.84 & 15.53 & 15.53 & 15.38 & 15.97 & 15.53 & 15.13 & 15.13 & 15.53 \\
\hline $22: 10$ & 9941 & 14.07 & 2.95 & 13.25 & 14.25 & 12.66 & 15.53 & 12.58 & 13.38 & 15.38 & 15.53 \\
\hline $23: 10$ & 9941 & 14.94 & 1.86 & 13.68 & 14.35 & 15.54 & 15.38 & 14.39 & 15.13 & 15.53 & 15.54 \\
\hline
\end{tabular}




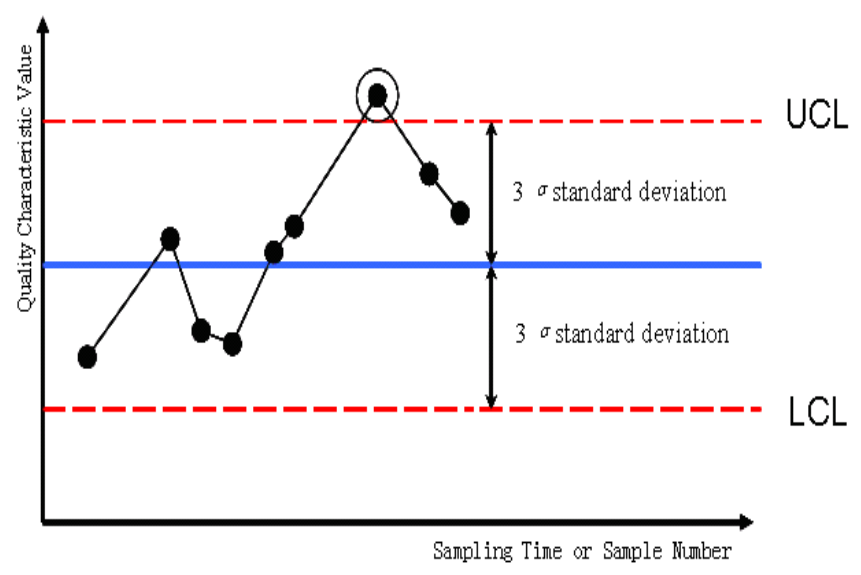

Figure 1. Simple Control Chart
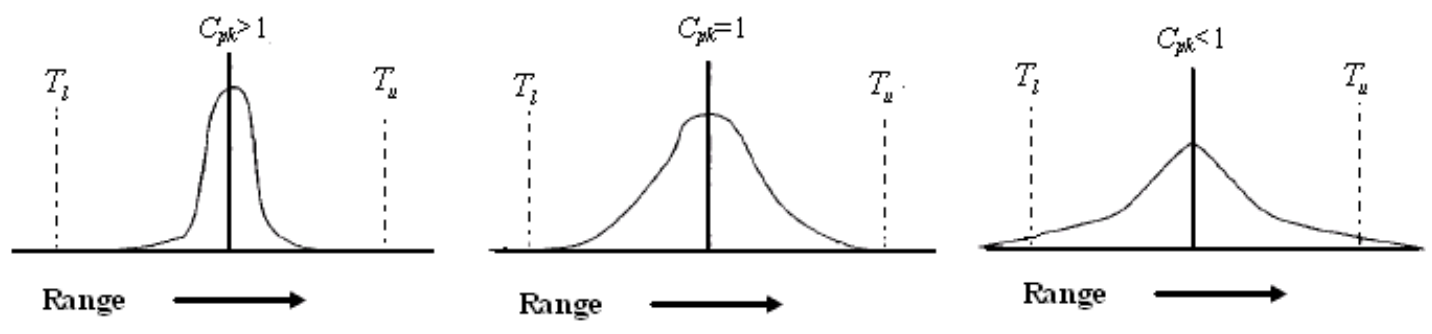

Note that $T_{l}$ denotes the upper limit of specification and $T_{u}$ denotes the lower limit.

Figure 2. Characteristic Curve of $C_{p k}$

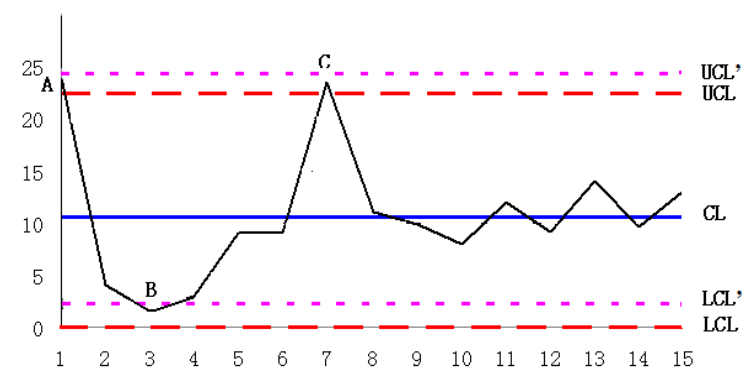

Figure 3. Non-corrected \& Corrected Control Chart

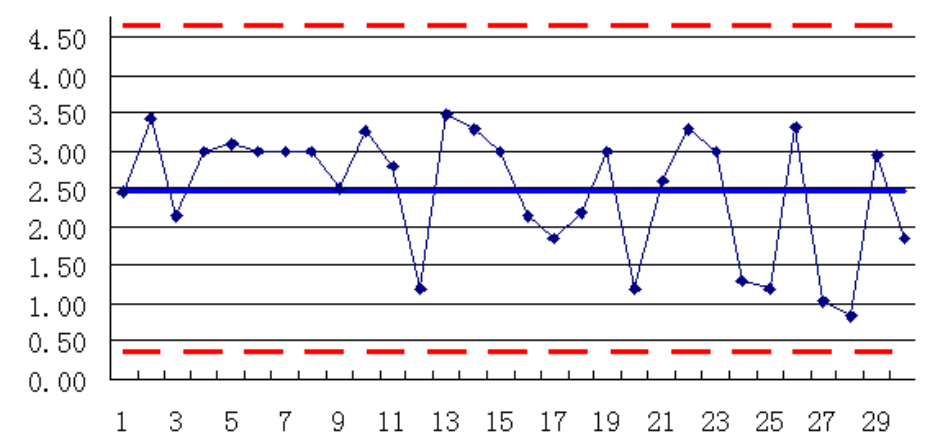

Figure 4. $R$ Control Chart 


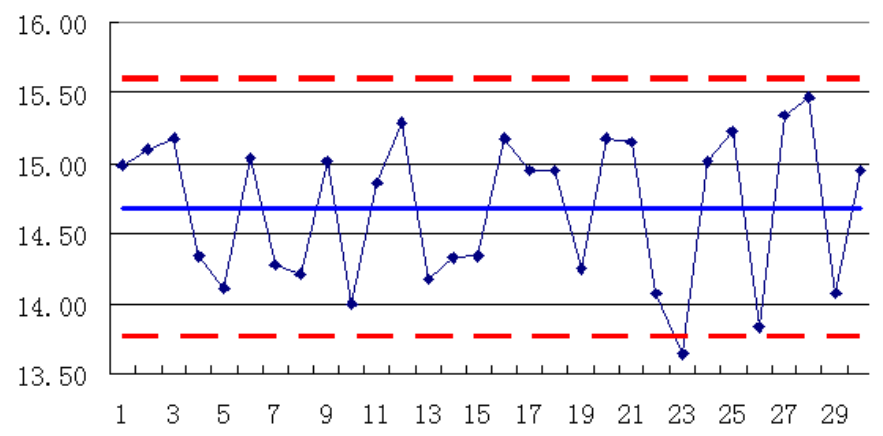

Figure 5. Non-corrected $\bar{X}$ Control Chart

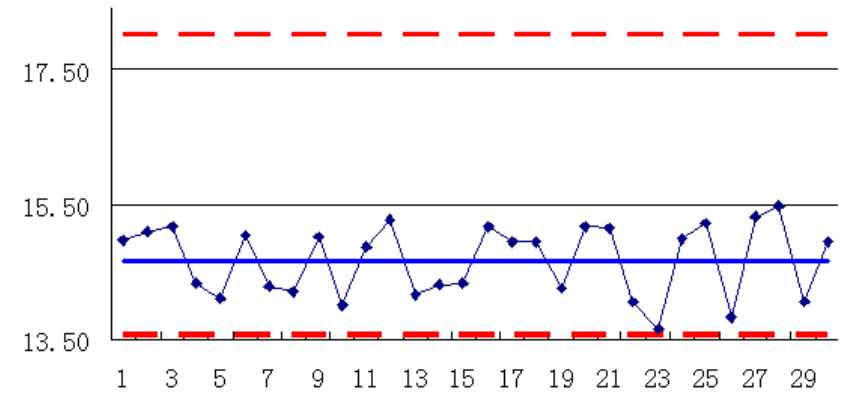

Figure 6. Corrected $\bar{X}$ Control Chart 